\title{
Wechselrede. Rhetorische Anmerkungen zur europäischen Brieftheorie
}

Le dialogisme dans les théories de la rhétorique épistolaire en Europe

Dialogism in theories of epistolary rhetoric in Europe

\section{Gert Ueding}

\section{(2) OpenEdition}

\section{Journals}

Édition électronique

URL : http://journals.openedition.org/ceg/826

DOI : $10.4000 /$ ceg. 826

ISSN : 2605-8359

Éditeur

Presses Universitaires de Provence

Édition imprimée

Date de publication : 30 mai 2016

Pagination : 21-34

ISBN : 979-10-320-0067-0

ISSN : 0751-4239

\section{Référence électronique}

Gert Ueding, «Wechselrede. Rhetorische Anmerkungen zur europäischen Brieftheorie », Cahiers d'Études Germaniques [Online], 70 | 2016, Online erschienen am: 17 Dezember 2017, abgerufen am 26 November 2020. URL : http://journals.openedition.org/ceg/826 ; DOI : https://doi.org/10.4000/ceg. 826 


\title{
Wechselrede
}

\section{Rhetorische Anmerkungen zur europäischen Brieftheorie}

\author{
Gert UEDING \\ Eberhard Karls Universität, Tübingen
}

Von europäischer Briefkultur sprechen, das heißt von der Rhetorik sprechen, auch wenn das Bewusstsein dieser Zuständigkeit durch eine oberflächliche Lektüre von Gellerts epistolographischer Reform verloren gegangen ist. Dessen Distanzierung von Rhetorik bezog sich auf den Stylus Curiae, der seit dem Mittelalter die Briefstellerei dominierte. Gellert selber befindet sich aber ganz in rhetorischer Überlieferung, in seinem Kampf gegen die Curialstil-Rhetorik bediente er sich der klassischen Quellen; er zitiert Cicero und Quintialian und bringt allenfalls mit seiner Betonung des Anmutig-Freundlichen einen eigenen Akzent in die Gattungsgeschichte. Im übrigen hält er sich sowohl was das prinzipielle Verständnis wie auch die einzelnen Empfehlungen und die Methode der Verbesserung der deutschen Briefkultur betrifft an seine antiken Quellen.

Die unseres Wissens nach erste rhetorische Brieftheorie und damit erste Reflexion über diese Prosagattung überhaupt stammt von Artemon aus dem 1. Jahrhundert. Im Vorwort seiner Aristoteles - Briefausgabe nennt er den Brief die eine Hälfte eines Dialoges, den Briefwechsel ein Gespräch unter Abwesenden. Soviel jedenfalls ist überliefert, auch wenn uns die Ausgabe selber nicht erhalten geblieben ist. ${ }^{1}$

Dass ausgerechnet des Aristoteles Briefe Anlass zu brieftheoretischen Überlegungen gegeben haben, ist kaum zufällig. Dessen „Rhetorik“, eines der grundlegenden Theoriewerke der Rhetorik, baut ihrerseits auf Errungenschaften der sophistischen Rhetorik, insbesondere des Protagoras, auf. Der Philosoph und Rhetor aus Abdera (etwa 485 - 415), der auch als demokratischer Verfassungsrechtler tätig war und als Autor des Homo-mensura-Satzes bis heute immerhin bekannt geblieben ist, hat die wesensmäßige Bindung der Rede an den Dialog zum Prinzip der Rhetorik erhoben - und zwar in seiner ganzen Vielfalt vom Lehrdialog bis zum Streitgespräch. Aristoteles, der seinem Vorgänger nicht sehr viel Gerechtigkeit widerfahren läßt, nimmt das dialogische Prinzip in seine Rhetorik auf. Er bezeichnet sie gleich im ersten Satz seines Buches zwar als „Gegenstück zur Dialektik“2, die methodisch dem Verlauf von Frage

1. Vgl. W.G. Müller: Artikel „Brief“, in Gert Ueding (Hrsg.), Historisches Wörterbuch der Rhetorik, 12 Bde, Tübingen, de Gruyter, 1992 ff., Bd. 2, 1994, Spalte 67.

2. Aristoteles, Rhetorik, übers. von Hans G. Sieveke, München, W. Fink, 1980 (1354ª), S. 7. 
und Antwort folgt, also dialogisch vorgeht, setzt aber das Strukturelement des Miteinanderredens in seine Konzeption ein, die immer im Bezug zu seinem Polis - Modell gelesen werden muss. In ihm ist jeder Bürger auch der mögliche Staatsmann und dieser wieder der mögliche Bürger, so dass jeder „abwechselnd

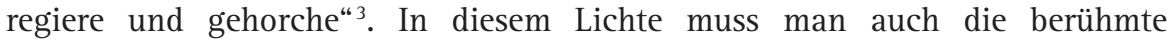
Rededefinition im 3. Kapitel des 1. Buches der „Rhetorik“ lesen. Ihm folgend stehen die drei an einer Rede beteiligten Instanzen, nämlich Redner, Redegegenstand und Adressat so zueinander, dass der Adressat die ganze Ausrichtung der Rede bestimmt. ${ }^{4}$ Das Publikum, so wie Aristoteles es sich wünscht und heranbilden will (für das reale Publikum findet er gelegentlich sarkastische Bezeichnungen) wird hier und an anderen Stellen seines Buches nicht als passiv empfangendes Auditorium verstanden, sondern als eines, das mitdenkt, miturteilt, also aktiv am Redegeschehen teilnimmt. Offensichtlich soll, alles in allem genommen, jeder Bürger die Rollen des Redners und des Zuhörers in seiner Person verkörpern: der Redner Mitzuhörer und der Hörer Mitredner sein.

Wir haben hier das Modell des korrespondierenden Wechsels von Sprecher und Adressat, wie es die europäische Briefrhetorik bis in die Moderne bestimmen wird. Werfen wir, diesen Punkt betreffend, noch einen kurzen Blick auf die römische Fortführung. Cicero, der für die Briefkultur des Humanismus zur wichtigsten Berufungsinstanz wird, übernimmt das Gesprächskonzept jedenfalls im Genus des Privatbriefs, den er „eine Wechselrede unter Freunden in Abwesenheit ${ }^{“ 5}$ nennt. Bei ihm findet sich auch ein darüber hinaus gehender Gedanke, der in der Folgezeit bedeutsam wird. Er skizziert nämlich eine Gattungsunterscheidung, deren triadische Form dem Schema seiner Rhetorik entstammt. Die bloße Tatsachenmitteilung findet sich in der untersten Kategorie; die ernste, bedeutungsvolle, politisch entscheidende Briefrede (genus severum) steht an der Spitze; zwischen beiden Extremen angesiedelt ist der persönliche, private, auch scherzhafte Brief (genus familiare), dem Ciceros größte Aufmerksamkeit gilt und in dem die dialogische Form ihre höchste Ausprägung erreicht. Ersichtlich spiegeln sich hier die drei Klassen rhetorischer Überzeugungsmittel: die argumentativ - sachliche auf der einen, die affektisch bewegende, erhabene auf der anderen Seite. Dazwischen aber die unterhaltsame, freundliche, liebenswürdige, der humanitas, der Mitmenschlichkeit, verpflichtete Weise der Beeinflussung.

Es hat in der Rhetorik der Briefsteller später eine bis zur Unübersichtlichkeit gehende Differenzierung von Briefgattungen und Brieftypen gegeben, die oftmals vor allem zeremonielle Bedürfnisse befriedigte. Ciceros Dispositionsschema machte nicht nur den Vorzug der Einfachheit für sich geltend, es bildete auch den Grundriss für mehrere Ableitungen wie die emotionale Wirkung (von neutral bis

3. Aristoteles, Politik, in Aristoteles, Philosophische Schriften, 6 Bde, Bd. 4, übers. von E. Rolfes (1252 a 16), Hamburg, Felix Meiner, 1995, S. 1.

4. Aristoteles, Rhetorik $\left(1358^{\mathrm{ab}}\right)$, S. $20 \mathrm{f}$.

5. Cicero, Ad familiares 2, 4, zit. nach Müller, „Brief“, Spalte 67 f. Vgl. auch Klaus Thraede, Grundzüge griechisch-römischer Brieftopik, München, Beck, 1970. 
heftig) oder schließlich für die Dreistil - Lehre, die auch auf den Brief übertragen wurde. Für das schlichte Informieren galt der niedere, schmucklose Stil, für den persönlichen, freundschaftlichen Brief der mittlere, sehr abwechslungsreiche und lockere Stil (natürlich ungebunden, wie Quintilian ergänzt) ${ }^{6}$, für den bedeutenden, würdevollen, achtunggebietenden Brief der hohe, erhabene Stil. Die Briefsteller des Mittelalters ergänzten, dem feudalen Ständestaat entsprechend, die Dreistillehre mit dem sozialen Status des Adressaten. Auf fatale Weise folgenreich bis in die Neuzeit, wenn man an den offiziellen Schriftverkehr der fürstlichen Ämter und Kanzleien denkt.

Wie schon angedeutet galt Ciceros größtes Augenmerk dem Freundschaftsbrief. Das hatte mehrere Gründe. Er eröffnete den Schreibern die größten Freiheiten, ohne aber nun alles in ihr individuelles Belieben zu stellen und Willkür an die Stelle von Form zu setzen. Mit Grund ergänzt Quintilian, dass „auch die ungebundene Form bestimmte ihr eigene und vielleicht gar noch schwierigere Rhythmen"7 besitze. Eine Kompliziertheit, die dem Meister wiederum einen eigenen Reiz bedeutete. Ein weiterer Vorzug dieses „genus familiare“ bestand in seiner Gesprächigkeit. Der dialogische Charakter äußert sich, das war allen Brieftheoretikern seit Artemon klar, zuletzt zwar doch als Simulation: der Gesprächspartner ist abwesend, er äußert sich schriftlich, und die Wechselreden treffen zeitversetzt bei den Korrespondenten ein. Aber der private, persönliche Brief kann die Simulation kunstreich ausbauen und so die Glaubwürdigkeit der Gesprächsfiktion steigern: durch vertraute Anrede, steten Bezug auf den Partner, Vorwegnahme seiner Reaktionen, Gefühle, Gedanken - kurz, durch seine fiktive Vergegenwärtigung im Raum des Miteinandersprechens.

Wir treffen hier auf einen Grundzug der europäischen Briefkultur. Sie geht nicht vom offiziellen Brief der Ämter oder vom Informationsbrief der Institutionen und ihrer Repräsentanten aus, sondern vom persönlichen, auf das Gefühlsleben der Korrespondenten aufgetragenen, in ihm lebendig werdenden Brief. Auch er hat seine Ordnung, seine Disposition, seinen Stil, seine Tonarten, doch seine Lizenz ist, verglichen mit anderen Prosaformen weitaus größer und mehr vom Geschmack der Autoren, als von der Autorität einer Gattungsregel abhängig. Diesem eigentümlichen Changieren zwischen Ordnung und Freiheit, Regel und Lizenz ist es auch zu verdanken, dass der persönliche Brief schon in der Antike eine Allianz mit theoretischer Verbindlichkeit einging - man braucht nur an Ciceros Schrift „Brutus“ zu denken, in der er die Geschichte der römischen Beredsamkeit darstellt, und die mit einer Klage über den Tod eines Freundes beginnt, oder an sein Buch „De re publica“, dem er, wir trotz des fehlenden Beginns erschließen können, einen Brief an seinen Bruder voransetzte, oder an die Episteln des Horaz, die seine berühmte „Ars poetica“ enthalten, oder an die pseudonym überlieferte Schrift „Vom Erhabenen“, die der Autor in Form eines Briefes an einen Freund eröffnete - die Beispiele lassen sich leicht vervielfachen.

6. Marcus Fabius Quintilianus, Ausbildung des Redners, hrsg. und übers. von H. Rahn, 2 Bde, Darmstadt, Wissenschaftliche Buchgesellschaft, 1975 (9, 4, 19), Bd. 2, S. 373.

7. Ibid., (9, 4, 20), S. 373. 
Sie verweisen uns nebenbei auf eine weitere Dimension des Briefes und seiner rhetorischen Verfassung: als Ursprungsort einer neuzeitlichen literarischphilosophischen Gattung, des Essays.

Doch zurück zum Hauptpunkt. Seit der Brief Gegenstand theoretischer Reflexion ist, gehört er in die Zuständigkeit der Rhetorik, die ihn, besonders in seiner persönlich-privaten Ausprägung, als Derivat des Gesprächs, nicht etwa der monologischen Rede, auffasste und entsprechend kultivierte. Die Briefrhetorik ist damit auch ein Zeugnis für das ausgesprochen dialogische Prinzip der Redekunst, ${ }^{8}$ wie es von Protagoras grundgelegt, von Aristoteles, Cicero und Quintilian aufgegriffen und differenzierend weiterentwickelt wurde. Wobei bezeichnenderweise Cicero sein rhetorisches Hauptwerk „De oratore“ (55 v. Chr.), nach Manfred Fuhrmanns Urteil „die bedeutendste Darstellung der Rhetorik, die die Antike hinterlassen hat" ${ }^{9}$, auch literarisch in Form eines Dialogs verfasst hat. Die Rede vom Briefgespräch wurde zum Topos, der im Humanismus ebenso weitergereicht wurde wie in der Aufklärung und selbstverständlich baut auch Gellert seine Reform des Briefes auf diesem Grundsatz auf: Der Brief „vertritt doch die Stelle einer mündlichen Rede, und deswegen muß er sich der Art zu denken und zu reden, die in Gesprächen herrscht, mehr nähern als einer sorgfältigen und geputzten Schreibart. Er ist eine freye Nachahmung des guten Gesprächs.“ ${ }^{10}$

So hätte auch Cicero schreiben können, wie überhaupt das Ideal der Natürlichkeit sich dem Rückgang zu den antiken Quellen verdankt, keine originäre Errungenschaft des 18. Jahrhunderts ist. Gellert und seine Nachfolger nehmen also nicht Abschied von der Briefrhetorik, vielmehr setzen sie an die Stelle einer überregulierten, am Curialstil orientierten und feudal instrumentierten Briefstellerei eine neue Rhetorik, die sich vor allem an Cicero und der humanistischen Briefkunst ausrichtete. Darin ist der Gesprächstopos keine folgenlose Referenz, er entspricht einem Grundzug der Epoche. „Der Mensch ist ein zahmes und geselliges / nicht aber ein wildes noch zur Einsamkeit geschaffenes Thier. Der Grund aller Gesellschafften ist die Conversation." ${ }^{11}$ Das ist Christian Thomasius in seinem „Kurtzen Entwurf der politischen Klugheit“ (1710). Im großen Bogen, am Ende des Jahrhunderts, wird ihm Christian Garve den Erfolg des Konzepts bestätigen:

Selbst da, in etwas spätern Zeiten, die eigentliche Wissenschaft oder die Philosophie entstand: erschien sie zuerst nur unter der Gestalt einer gesellschaftlichen Unterhaltung. Für die Vernunft hatten die Griechen keinen andern Namen, als den der Rede; wissenschaftliche Untersuchungen anstellen hieß bei ihnen, sich über die Gegenstände derselben unterreden;

8. Vgl. Gert Ueding, „Dialogrhetorik“, in Gert Ueding / Gregor Kalivoda (Hrsg.), Wege moderner Rhetorikforschung, Berlin/Boston, de Gruyter, 2014, S. 703-714 und Manfred Fuhrmann, Die antike Rhetorik, München/Zürich, Artemis \& Winkler, 1984.

9. Fuhrmann, Die antike Rhetorik, S. 52.

10. Christian Fürchtegott Gellert, Die epistolographischen Schriften, Faksimiledruck nach den Ausgaben von 1742 und 1751, hrsg. von R.M.G. Nickisch, Stuttgart, Metzler, 1971, S. 3.

11. Christian Thomasius, Kurtzer Entwurf der politischen Klugheit, Frankfurt und Leipzig, 1710, S. 108. 
und zufolge ihres ältesten Namens ist die Logik nichts anders, als die Kunst eines gelehrten Gesprächs. ${ }^{12}$

In Dialogform erscheinen daher selbst schwierige philosophische Darlegungen (Moses Mendelssohns z.B.), neben ihn tritt der Brief als eine der beliebtesten Darstellungsformen; Schillers Briefe über die ästhetische Erziehung mögen als prominentestes Beispiel dienen.

Nun macht Gellert eine Einschränkung, die für den Briefessay sowieso gilt, der sich an den unbekannten Leser richtet, die er aber moderat auch im persönlichen Brief beachtet wissen will: er spricht von einer Annäherung der Denk- und Schreibart des Briefes an das Gespräch, also nicht etwa von einer Kopie. Man braucht nicht lange zu rätseln: er greift hier ersichtlich zurück auf das rhetorische imitatio - Konzept, das niemals sklavische Nachahmung bedeutet hat, sondern die Übertragung eines Musters in einen anderen Kontext, hier sogar aus der geselligen Realität in das schriftliche Medium des Briefes. Warum, so kann man fragen, soll dann der Brief sich überhaupt am Gespräch orientieren, wenn er sich doch von ihm entfernt, wenn er stilisiertes, von Redundanzen, Weitschweifigkeiten, Irrtümern und sprachlichen Fehlern befreites Produkt der Schreibkunst handelt? Weil es darauf ankommt, die, um es mit Karl Löwith zu sagen, „Strukturmomente des Miteinandersprechens“ ${ }^{13} \mathrm{zu}$ erhalten in der brieflichen Übertragung, und das heißt im Wesentlichen, persönliche Zuwendung und Anrede, sowie der Tendenzinhalt auf Erwiederung, der auch eine Haltung bedeutet: im Brief, wie im Gespräch verhalte ich mich fragend, suchend, auch versuchend und selbst meine Behauptung ist auf Gegenbehauptung angelegt. Wilhelm von Humboldt hat die dialogische Eigenart der Sprache selber zuerkannt:

Besonders entscheidend für die Sprache ist es, dass die Zweiheit in ihr eine wichtigere Stelle, als irgendwo sonst einnimmt. Alles Sprechen ruht auf der Wechselrede, in der, auch unter mehreren, der Redende die Angeredeten immer sich als Einheit gegenüberstellt. Der Mensch spricht, sogar in Gedanken, nur mit einem andren, oder mit sich, wie mit einem andren, und zieht danach die Kreise seiner geistigen Verwandtschaft, sondert die, wie er, Redenden von den anders Redenden ab. ${ }^{14}$

Wenn der Brief derart aus dem dialogischen Wesen der Sprache selber lebt, ist seine Kultivierung gleichbedeutend mit der der Kultivierung der Sprache. Auch an dieser Stelle greift Rhetorik ein, und das schon in ihren Ursprüngen. Protagoras hatte in seiner als mythologische Erzählung formulierten Kulturentstehungstheorie der Sprache die entscheidende Rolle zugeteilt und Aristoteles definierte bekanntlich den Menschen als zoon logon echon, als das Wesen das Sprache hat. Deren Verbesserung, Verfeinerung beschreibt also den Königsweg zur Vervollkommnung der Sitten und Umgangsformen. Gellert, jeder Künstlichkeit und Geziertheit abhold, versteht das nicht anders.

12. Christian Garve, Über Gesellschaft und Einsamkeit, 2 Bde, Breslau, 1800, Bd 1, S. 19.

13. Karl Löwith, Das Individuum in der Rolle des Mitmenschen, Darmstadt, Verlag Karl Alber, 1969, S. 109.

14. Wilhelm von Humboldt, „Über den Dualis“, in ders., Schriften zur Sprache, Frankfurt a.M., Zweitausendeins, 2008, S. 129. 
Allein wer sieht nicht, dass wir im Briefschreiben in viele Fehler verfallen würden, wenn wir ohne Unterscheid (sic!) die Sprache des Umgangs nachahmen wollten? Unsre Schreibart würde sehr oft unverständlich und schmutzig, oder gezwungen, platt, weitläuftig und gemein werden, wenn wir ohne Ausnahme von bürgerlichen und häußlichen Angelegenheiten in Briefen so reden wollten, wie die Niedrigen, die Vornehmen, im gemeinen Leben davon zu sprechen pflegen. Hier geht also der Brief von dem Gespräche ab. ${ }^{15}$

In der „Wiederbringung der teutschen Beredsamkeit“"16 hatte einst Leibniz eine Bedingung für die Renaissance deutscher Wissenschaften und Künste gesehen, auch Gellert begreift seine Reform des deutschen Briefstils im großen Zusammenhang einer allgemeinen Geschmacksverbesserung. Sie kommt ohne Kunstfertigkeit, ohne Geschick und Gelehrigkeit nicht aus. Ein Zugeständnis, das er sogleich zu entschärfen sucht und damit zu einem von langher aus der Antike vertrauten Prinzip greift:

\footnotetext{
Zuweilen kostet eben das Leichte, das Natürliche [...] die meiste Mühe, und gefällt doch dem Leser am Ende aus dem Grunde, weil es keine Mühe gekostet zu haben scheint. Man hat alle Arbeit, alle Kunst versteckt. ${ }^{17}$
}

Vom artem celare - Prinzip hatte die lateinische Rhetorik gesprochen. So können wir die Natürlichkeit des Briefstils getrost ein kunstvolles Erzeugnis nennen, das immer auch ein Stück Verfremdung enthält. Hier liegt nun eine Gefahr verborgen, auf die besonders nachdrücklich der Freiherr Knigge hingewiesen hat. Der Brief ist Bestandteil des geselligen Umgangs, er ist Teil der „gesellschaftlichen Beredsamkeit" ${ }^{18}$ wie Knigge seine Umgangskunst nannte, gerät aber in diesem Kontext seinem Vorbild, dem lebendigen Gespräch, gegenüber in einen Nachteil. Im 33. Abschnitt des 1 . Teils seines „Umgang mit Menschen“ bemerkt sein Autor:

Briefwechsel ist schriftlicher Umgang; fast alles, was ich vom persönlichen Umgange mit Menschen sage, leidet Anwendung auf den Briefwechsel. Dehne also Deinen Briefwechsel, so wie Deinen Umgang, nicht über Gebühr aus. Das hat keinen Zweck, kostet Geld und ist Zeitverderb. Sei ebenso vorsichtig in der Wahl derer, mit denen Du einen vertrauten Briefwechsel anfängst, als in der Wahl Deines täglichen Umgangs und Deiner Lektüre. [...] Vorsichtigkeit ist im Schreiben noch weit dringender als im Reden zu empfehlen, und ebenso wichtig ist es, mit den Briefen, die man erhält, behutsam umzugehen. Man sollte es kaum glauben, was für Verdruß, Zwist und Mißverständnis durch Versäumung dieser Klugheitsregel entstehen können. Ein einziges hingeschriebenes unauslöschliches Wort, ein einziges aus Unachtsamkeit liegengebliebenes Papier hat manches Menschen Ruhe und oft auf immer den Frieden einer Familie zerstört. ${ }^{19}$

Was im Gespräch sofort zurückgenommen, erläutert, entschuldigt oder aufgeklärt werden kann, fixiert der Brief und stellt es womöglich noch aus. In dieser Perspektive der Gefährdung des gesellschaftlichen oder geselligen Zusammenhalts gewinnt die rhetorische Kultivierung des Briefes einen weiteren

15. Gellert, Die epistolographischen Schriften, S. 6.

16. Gottfried Wilhelm Leibniz, Politische Schriften, 2 Bde, hrsg. von H. H. Holz, Frankfurt a.M., Europäische Verlagsanstalt,1966, Bd. 2, S. 70.

17. Gellert, Die epistolographischen Schriften, S. 14.

18. Adolph Freiherr von Knigge, Über den Umgang mit Menschen, hrsg. von G. Ueding, Frankfurt a.M., Insel, 1977, S. 49.

19. Ibid., S. $55 f$. 
eminent praktischen Sinn. Wie der Brief Träger der Verständigung und des gemeinschaftlichen Lebens sein kann, so kann er es auch stören, im schlimmsten Falle zerstören - ja sogar sein Ausbleiben kann solche Wirkung haben. Literatur und Theater haben sich eben diese entfremdende Wirkungsmöglichkeit zunutze gemacht und dramatische Konflikte aus ihr gewonnen. Eine ganze Literaturgattung, in der dieser Gebrauch des Briefes notorisch wurde, beginnt auch schon mit einem entsprechenden Eklat: Edgar Allan Poes „Der entwendete Brief" von 1844.

Die verfremdende Wirksamkeit des Briefdialogs hat jeder Briefschreiber im eigenen Procedere erfahren. Es erschien dann leichter, sich im Briefe zu äußern als von Angesicht zu Angesicht. Auch das Gegenteil kommt vor, der Schreiber verzweifelt und legt das Schriftstück manchmal für immer beiseite - aber der Umstand ist seltener und in der Regel auf aussichtslose Fälle der Kommunikation beschränkt. Die größere Mühelosigkeit ist nun nicht nur ein Resultat der zeitlichen und räumlichen Distanz der Korrespondenten, obwohl sie mitspielen. Autor und Adressat tauschen nicht allein diese ihre Rollen im Laufe eines Briefwechsels, sie nehmen darüber hinaus Eigenschaften und Prägungen an, die einem der, wie gesehen, ältesten Kennzeichen des Briefes, nämlich persönlicher Ausdruck zu sein, zweifelhaft macht, jedenfalls modifiziert.

Der erste Rollentausch ist schon aus der Aristotelischen Rhetorik vertraut: Jeder Korrespondent ist, dem Sprecher -/ Hörer - Wechsel entsprechend, abwechselnd Schreiber und Adressat von Briefen. Doch indem der jeweilige Briefautor den Empfänger auf Erwiderung hin anschreibt, mit ihm etwas teilen (Mitteilung) will, bemüht er sich, so weit wie möglich, dessen Perspektive einzunehmen: wie wirkt das, was ich mitzuteilen habe, auf den anderen, wie formuliere ich verständnisvoll, wie vermeide ich ein Missverständnis? Meine Selbstaussprache geschieht also immer schon mit Rücksicht (oder ihrem bewußten Vermeiden) auf meinen Briefpartner, sie ist immer auch Ansprache. Doch damit nicht genug. Ich nehme darüber hinaus weitere Rollen ein, die sich nach meiner Absicht und nach den Erwartungen meines brieflichen Gesprächspartners richten. Jede dieser Rollen kann stabil sein (die Rolle des Vaters in Briefen an den Sohn, die Rolle des Geschäftsfreundes für den Kompagnon, die Rolle der Geliebten für den Freund usw.), aber auch innerhalb eines Briefwechsels, ja sogar eines einzelnen Briefes ausgetauscht werden. Das dialogische Prinzip wird dadurch nicht etwa geschwächt, sondern verstärkt, und dieser rhetorischen Erfahrung verdanken wir denn auch die erste Rollentheorie in der Geschichte. Sie erwächst aus der Gerichtsrhetorik:

Der Weg vom Theater zum Gericht war besonders bequem: hier wie dort wurde ja im prägnanten Sinne gehandelt (agere, actio, actor); hier wie dort gab es feste Rollen, und hier wie dort konnte nur aus deren gemeinsamer, wechselseitig Bezug nehmender, ineinandergreifender Tätigkeit das Ganze - vom ersten bis zum füften Akt im Drama, von der Anzeige oder Ladung bis zum Urteil im Prozess - hervorgehen. ${ }^{20}$

20. Manfred Fuhrmann, „Persona, ein römischer Rollenbegriff“, in ders., Brechungen. Wirkungsgeschichtliche Studien zur antik-europäischen Bildungstradition, Stuttgart, Klett-Cotta, 
In seinem Dialogbuch „De oratore“ läßt Cicero die Gesprächsfigur Antonius von sich sagen: „So übernehme ich [...] vollkommen unparteiisch drei Rollen in einer einzigen Person, die meine, die des Gegners und die des Richters.“" ${ }^{21}$ Der lateinische Text nennt die drei Rollen tris personas, und persona ist der Begriff, den Cicero für seine Rollentheorie fruchtbar gemacht hat. Manfred Fuhrmann erläutert ihn: „Wie immer man persona [...] übersetzen mag: gemeint ist stets der Träger einer sozialen Rolle, nicht die absolute Person, das Individuum." ${ }^{22}$ Im ersten Buch von „De officiis“ hat Cicero die Ansätze theoretisch und mit Beispielen versehen weiter ausgeführt. Die Herkunft von persona aus der Theatersphäre, wo der Terminus „Maske“ und dramatische Rolle bezeichnet, bleibt dabei immer erhalten und weitet sich aus zur Vorstellung vom Welttheater, dem theatrum mundi. Die Metapher gewinnt ihre Gestalt und Wirkung zwar erst in der Neuzeit, steht aber als Gedanke schon hinter Ciceros Rollentheorie, wenn er die „Art der Lebensgestaltung“, die „Lebensart“ als Ergebnis einer Nachahmung derer beschreibt, „die nachzuahmen uns gut scheint“" ${ }^{23}$. Beispiele aus Geschichte und Literatur (Cato, Aias, Odysseus) bekräftigen die Übertragung. „Unter den Griechen war Sokrates gewinnend, witzig, von launigem Gesprächston und in jedem Dialog ein Meister der Verstellung [...].“24

Keineswegs also ist es nur der Gerichtsredner, der eine Vielzahl von Rollen verkörpert. Cicero ist vielmehr der sehr realistischen Ansicht, dass wir alle, auch wenn „ein jeder an seinen Eigenschaften festhalten“ ${ }^{25}$ soll, in unseren Handlungen ein ganzes Ensemble von Rollen einnehmen. Das gelte auch für Gespräche, fügt er hinzu, in ihnen erreiche zum Beispiel „gerade ein gerissener Mensch“ dadurch seine Ziele, dass „er einer von vielen zu sein scheint“26. Kein Zweifel, dass Cicero das Rollenverständnis des Menschen gemäß seiner Überzeugung von der Ubiquität der Rhetorik allgemein auf die Lebenspraxis überträgt, und der Brief in diesem Zusammenhang nicht etwa eine Ausnahme macht, auch dann nicht, wenn er dem genus familiare angehört.

Dieser Gedanke ist einer doppelten Auslegung fähig, die sich auch in der Geschichte des Briefes und der Briefstellerei abbildet. Einmal nämlich als ein den Umständen und Wirkungsintentionen folgendes Rollenspiel, hinter dem die Identität der Korrespondenten zurücktritt; das Zeremonienwesen in der Briefstellerei des 16. und 17. Jahrhunderts mag dafür als Exempel dienen. Ein andermal aber als occasionelles, situatives Umkleiden in andere Rollen unter der Bewahrung der eigenen Natur, so etwa wäre Gellerts Position zu beschreiben. Natürlichkeit, Ausdruck der eigenen Natur, ist seine oberste Briefregel, die

1982, S. 27.

21. Cicero, De oratore. Über den Redner, lat./deutsch, übers. u. hrsg. von H. Merklin, Stuttgart, Reclam, 1976, S. 271 (II, 102).

22. Fuhrmann, „Persona“, S. 30.

23. Cicero, De officiis. Vom pflichtgemäßen Handeln, lat./deutsch, übers. u. hrsg. von H. Gunermann, Stuttgart, Reclam, 1976, S. 103 (I, 118).

24. Ibid., S. 95 (I, 108).

25. Ibid., S. 97 (I, 110).

26. Ibid., S, 97 (I, 109). 
aber nicht verhindern darf, wenn nötig dankbare Haltung und Ehrerbietung, Bescheidenheit und Lebhaftigkeit an den Tag zu legen, weil das dem Adressaten, einem Gönner und Wohltäter, angemessen ist. ${ }^{27}$ Überdies war Gellert, wie erwähnt, auch die Einsicht nicht verschlossen geblieben, dass Natürlichsein ein Kunstprodukt ist und damit eine eigene Maske, die jederzeit abgelegt werden kann.

Wenig Beachtung gefunden hat bisher, dass im unausweichlichen Rollenverhalten artistische Möglichkeiten verborgen sind und zudem die Lizenz gegeben ist, Erfahrungen und Gefühle auszusprechen, die sonst stumm geblieben wären. Es versteht sich, dass insbesondere Künstler und Schriftsteller, die Spezialisten kunstvoller Mimikry und phantastischer Sujets, sich des brieflichen Rollenspiels mit eigener Findigkeit bedienten.

Werfen wir im Vorübergehen den Blick auf zwei Beispiele. In beiden geht es darum, im Sinne der Narrentradition, im Schutze der Verkleidung heikle Wünsche oder Befürchtungen mehr oder weniger deutlich auszudrücken. Das erste führt zurück in die zweite Hälfte des 19. Jahrhunderts. Seit Juni 1867 hielt sich Wilhelm Busch auf Einladung der Bankiersfamilie Keßler sporadisch, aber auch über längere Zeiträume zwischen 1869 und 1872 in Frankfurt auf und bewohnte die leer stehende Kutscherwohnung im Hinterhause der Kesslers. Wenn er nach München oder ins heimatliche Wiedensahl verreist war, schrieb er Johanna Keßler, die ihn zwar unter ihrer kunstliebenden Fittiche genommen hatte, aber von ihm ersehnte intimere Kontakte nicht zuließ. Die Briefe spiegeln, metaphorisch verschlüsselt, diese verquere Situation, dass sich Busch dies erlauben durfte, ohne sogleich zur Ordnung gerufen zu werden, verdankte er zwei Kunstgriffen: er erfand zwei Brief - Rollen und benutzte für seine Gefühlserklärungen objektive Korrelate, etwa Natur- oder Wetterverhältnisse. „Liebe Tante“, oder „Meine liebe Tante“ oder „Meine liebe gute Tante“ lautet die Anrede und er verabschiedet sich mit „vielen“ oder gar „tausend herzlichen Grüßen“ von „Ihrem getreuen Onkel Wilhelm“. Wenn auch in späteren Briefen die Selbsternennung zum Onkel fehlt, seine Maske bleibt erhalten, weil die Adressierung der „Tante“ niemals aufgegeben wird. Am 12 Februar 1875 schreibt er ihr aus Wiedensahl:

\footnotetext{
Ihre freundlichen Zeilen und die der Kinder erhielt ich heut Mittag. - Sie vertraun dem milden Einfluß der Zeit. Wohl und gewiss! Aber doch, derweil wir wandeln, geht all das Gute, was wir nicht getan und all das Liebe, was wir nicht gedurft, ganz heimlich leise mit uns mit, bis dass die Zeit für dieses Mal vorbei. Es weht der Wind; das Schneegestöber hüllt mir Wald und Feld und Garten ein. Ich wollt ich wär ein Eskimo, säße hinten am Nordpol, tief unter der Schneekruste, tränke Leberthran und könnte mich wärmen, an Was ich möchte. / Bei Ihnen brennt’s Feuer im Kamin. Da säß ich auch recht gern. ${ }^{28}$
}

Eine doppelbödige, von der gebildeten Adressatin leicht zu deutende Briefrede, die einen Liebesbrief voller Resignation und Bitterkeit und verlorener Hoffnung

27. Gellert, Die epistolographischen Schriften, S. 16 f.

28. Wilhelm Busch, Sämtliche Briefe, 2 Bde, hrsg. von F. Bohne, Hannover, Wilhelm-BuschGesellschaft, 1968, Bd. 1, S. 127. 
kaschiert, und zwar im Schutze der Imitation eines Briefpartner-Verhältnisses von Tante und Onkel.

Das zweite Beispiel wähle ich aus der Korrespondenz einer Dichterin des 20. Jahrhunderts. Am 2. März 1932 schreibt Else Lasker-Schüler den ersten Brief an Feuerspeiender Berg:

Feuerspeiender Berg / Es sagte einer an dem Tag wo wir uns sahen, Ihr habet alles von den alten Meistern an Talent in Euch versammelt; der zweite sagte, Ihr seid ein Verstandesmensch, der mit dem Verstand malte und auch handelte. Und ich weiß nur, daß ich mit Euch einen Urwald aufbauen möchte und eine neue Prärie legen möchte und wilde Pferde bändigen möchte, die uns nachher sehr dankerfüllt sein werden, da wir sie, namentlich die Zebra, wie seltene Farrenblätter hüten wollen und bewahren. Denn Pferde der Prärie, feuerspeiender Berg, sind viel vornehmer wie Menschen. [...] Die kleine Feder schmücke Euch, feuerspeiender Berg, immerdar und allerwegen / Pampeia. ${ }^{29}$

Im Werk der Dichterin firmiert eine „Correspondenz mit einem bösen Indianer“ als „Indianerbriefe“ ${ }^{30}$; ihr Adressat ist wohl der Maler Gert Wollheim. ${ }^{31}$ Es sind nur neun Briefe, in denen die beiden Briefpartner eine eigene Phantasiewelt schaffen, zu der auch neue Identitäten gehören, eben „Feuerspeiender Berg“ und „Pampeia“. Für unser Thema von Interesse sind vor allem drei Eigentümlichkeiten dieses Briefwechsels. Die beiden Korrespondenten kennen sich nur von ferne, und Else Lasker-Schüler legt großen Wert auf diese Distanz zur alltäglichen Lebenswelt:

Aber wir, im Sternbild des Syrius, die wir uns Sternenschein senden, überhaupt uns nur im Schein begegnen können - erliegen von kleinster Tatsache des irdischen Lebens. ${ }^{32}$

Je entfernter diese „Tatsachen“ sind, umso freier kann sich die Gegenwelt des Rollenspiels entfalten. Darin wird der Brief zum Eintrittsbillet in ein Wunschdasein. Was geschieht dadurch mit den beiden Korrespondenten? Ihre Charaktere und Handlungen rücken aus dem Gewohnten, ihnen Vertrauten und sie lernen sich selber und den anderen von Seiten kennen, die sonst verborgen waren. Eine mitunter erschütternde Selbstbegegnung ist dann das Ergebnis. Drittens schließlich: auch der umgekehrte Effekt tritt ein. Aus der Perspektive der Briefrealität (denn eine - psychische - Realität ist auch sie) erscheint das ausserhalb ihrer übliche Leben blass, banal, gegebenenfalls falsch. Der Brief bewirkt in den beiden zitierten Fällen, bei Busch wie bei Lasker-Schüler, eine Ent - Täuschung im positiven Sinne und die Schuppen fallen von den Augen.

Die zitierten Beispiele illustrieren aber noch eine weitere Erfahrung brieflicher Kommunikation, die Nietzsche so schön als „Punsch der Briefstimmung““33 formuliert hat. Es geht um die affektische Qualität des Briefgespräches, die

29. Else Lasker-Schüler, „Indianerbriefe“, in Anne Overlack, Was geschieht im Brief. Strukturen der Briefkommunikation bei Else Lasker-Schüler und Hugo von Hofmannsthal, Tübingen, Stauffenburg Colloqium, 1993, S. 132-152, hier S. 32.

30. Ibid.

31. Overlack, Was geschieht im Brief, S. 155 f.

32. Lasker-Schüler, „Indianerbriefe“, S. 136.

33. Friedrich Nietzsche, Werke in drei Bänden, hrsg. von Karl Schlechta, München, Hanser, 1958, Bd. 3, S. 985. 
Schreiber und Empfänger auf unterschiedliche Weise erzeugen und erleben. In der Briefrhetorik spielt sie nur eine geringe Rolle, aber nicht weil sie etwa als nebensächlich gilt, sondern weil ihre Funktion sich von der Funktion der Gefühlsrede allgemein nicht unterscheidet. Für die emotionale Gestimmtheit oder Stimmungserzeugung spielt allerdings die Eigenheit des brieflichen Gesprächs: räumliche Entfernung und zeitliche Verschiebung eine durchaus eigenartige Rolle.

Um die daraus resultierenden Konsequenzen zu verstehen, ist es nützlich, sich der Funktionen zu vergewissern, die die Rhetorik den Affekten für das Herstellen von Überzeugung zuwies. Sehr klar hat dies bereits Aristoteles getan. Gleich zu Anfang des 2. Buches seiner auf Vorlesungen basierenden „Rhetorik“, in der er seine rhetorische Affektenlehre formuliert, umreißt er eindringlich das Kernproblem; die Stelle sei daher ungekürzt hierher gesetzt:

\begin{abstract}
Denn im Hinblick auf die Glaubwürdigkeit macht es viel aus - besonders bei den Beratungen und schließlich vor Gericht - ,daß der Redner in einer bestimmten Verfassung erscheine und daß die Zuhörer annehmen, er selbst sei in einer bestimmten Weise gegen sie disponiert, und schließlich, ob auch diese sich in einer bestimmten Disposition befinden. Daß der Redner nämlich in einer bestimmten Verfassung erscheine, ist besonders nützlich bei der Beratung, und dass der Hörer in einer bestimmten Weise disponiert sei, ist vorteilhafter bei Gerichtsverhandlungen; denn ein und dasselbe erscheint nicht in gleicher weise den Liebenden und Hassenden bzw. den Zornigen und denen in sanfter Gemütslage, sondern die Ansichten sind entweder ganz und gar oder hinsichtlich ihrer Gewichtigkeit verschieden: dem Liebenden nämlich erscheint der, über den er ein Urteil zu fällen hat, entweder gar nicht schuldhaft oder nur in geringem Maße, dem Hassenden dagegen umgekehrt. Ebenso erscheint demjenigen, der von Verlangen und Hoffnung erfüllt ist, das, was kommen soll, sofern es angenehm ist, als etwas, das wirklich kommt, und als etwas Gutes; bei dem aber, der gleichgültig und in verdrießlicher Stimmung ist, ist das Gegenteil der Fall. ${ }^{34}$
\end{abstract}

Ich will mich nun nicht in die Geschichte der rhetorischen Affektenlehre vertiefen und verlieren. Ihre Basis liegt in der anthropologischen Erkenntnis vom Menschen als eines ziemlich umfänglichen Triebwesens, das seine Affekte weder abschalten kann, noch dies auch tun soll. Den platonischen Fehler, „die Affekte [...] uns denkbar [zu] machen, d.h. sie [zu] leugnen und als Irrtümer des Intellekts [zu] behandeln" ${ }^{35}$ hat Nietzsche daher kritisch abgewehrt. Wie die Teilnehmer eines Gespräches zueinander stehen, in welcher emotionalen Disposition sie sich befinden, ist für den Verlauf der Unterredung, für ihr Gelingen oder Misslingen nicht etwa von nebensächlicher, sondern von entscheidender Bedeutung. Wir sprechen dann davon, dass die Art und Weise, wie man zueinander „gestimmt“ ist, den Dialog „bestimmt“ habe. Und tatsächlich erfahre ich solche Basisvorraussetzungen wesentlich durch die Stimme des oder der anderen und verrate meine eigene Disposition auf dieselbe Weise; auch der Körpersprache kommt dabei eine wichtige Rolle zu.

Wie ist das aber nun im Brief? Auch bei ihm sprechen wir vom „Ton“ eines Briefes; das ist natürlich „übertragene“ Redeweise, bezieht sich aber auf die Grundstimmung, die einen Briefwechsel, oder den Part eines der beiden

34. Aristoteles, Rhetorik, S. 83f. (1377 $\left.{ }^{\mathrm{b}}-1378^{\mathrm{a}}\right)$.

35. Nietzsche, Werke, Bd. 3, S. 871. 
Biefschreiber oder gar nur einzelne Briefe beherrschen. Gemeint ist jedes Mal die emotionale $\mathrm{Zu}$ - oder Abwendung, die Art des Gefühls der Briefpartner zueinander. Doch was im mündlichen Gespräch durch den unwillkürlichen Ausdruck der Stimme erzeugt wird, dieser Grundton, muss im simulierten Gespräch des Briefes artistisch, mithilfe rhetorischer Kunst, hervorgerufen werden. Und hier kommt nun die Inszenierung zum Zuge, die jeder Briefwechsel darstellt, ob Rollen und direkt oder indirekt verabredetes 'Drehbuch' nur leicht oder (wie im Beispiel der „Indianerbriefe“) sehr stark von der Alltagswelt der Schreiber abweichen. Deren wichtigste Mittel sind Figuren und Tropen, die man auch die „Sprache der Affekte“ ${ }^{36}$ genannt hat, und deren individueller Gebrauch der Situation sowie den persönlichen Absichten und Gefühlseinstellungen folgt. Dabei erleichtert die Entfernung das bildhafte Gefühlsbekenntnis wie im Falle Wilhelm Buschs, weil nicht gleich mit Reaktionen wie Verboten oder ambivalenter Zustimmung gerechnet werden muss; oder sie ermöglicht den zeitweisen Aufenthalt in einem phantastischen Wunschensemble wie es sich Else Lasker-Schüler ausgedacht hat und in das sie ihren Briefpartner hineinzieht. Der „Ton“ des Briefes oder Briefwechsels, obgleich unausgesprochen, entsteht als Resultante der ausgewählten Mittel und ist in jedem Satz spürbar: melancholisch bei Busch, spielerisch übermütig bei Else Lasker-Schüler.

Störungsfreiheit beim Vorbringen der eigenen Gesprächsbeitrages muß also als durchaus schöpferische Situation begriffen werden, die, anders als die des Schriftstellers (der mit seinem Werk auch Gefühle beim Leser oder Zuhörer hervorbringen will) durch den individuellen Bezug der Korrespondenten aufeinander eine intime Note gewinnt: der Liebesbrief ist dafür die augenscheinlichste Probe aufs Exempel. Doch ich erreiche den Partner mit meinen Gefühlen nur, wenn ich sie selber empfinde, weshalb - eine der ältesten rhetorischen Maximen erinnernd - Gellert vor übersteigerten und falschen Gefühlen warnt und auch für sie Natürlichkeit, also Echtheit fordert. Doch wie sich in diese Stimmung versetzen, wenn doch der Adressat fern und die Möglichkeit gegenseitigen Stimulierens in der augenblicklichen Situation fehlt? Die Antwort gibt eine weitere rhetorische Maxime: Man ruft Phantasiebilder in sich herauf, von Begegnungen und Einbildungen, von Wunschszenarien und aus der Lektüre früherer Briefe. Es sind bewegende Bilder (Imagines agentes hat sie die Rhetorik genannt), die den Vorzug haben, auch gleich in Sprache übersetzt werden zu können und damit den gleichen Zweck, die Gefühlsstimulierung beim Adressaten zu erreichen vermögen. „Ich streichliche Deine Hände und wenn wir in den Urwald gehen, küsse ich Dich. Ich küsse Dich.“ ${ }^{37}$ Selbst der unbeteiligte Leser spürt, wenn auch sehr viel schwächer, die Bildvorstellung, die Else Lasker-Schüler beim Schreiben solcher Sätze bewegte. Denn tatsächlich wirken Sie nur, wenn der andere nicht nur aus taktischen, also gewissermaßen vernünftigen Erwägungen auf das Spiel eingegangen ist, sondern auch seine

\footnotetext{
36. So zum Beispiel Friedrich Andreas Hallbauer, Anweisung zur verbesserten Teutschen Oratorie, Jena 1725, ND Kronberg/Ts, Scriptor, 1974, S. 490.

37. Lasker-Schüler, „Indianerbriefe“, S. 142.
} 
Gefühle antworten. Der Adressat ist tatsächlich schon auf dieser Ebene, der Ebene des Gefühlsaustauschs, immer auch Mitautor - oder eben nicht, und dann scheitert der Briefwechsel. So erging es den „Indianerbriefen“. Als sich die beiden Korrespondenten im August 1932 im Romanischen Café, dem Berliner Künstlertreffpunkt, sahen, überraschte Wollheim seine Partnerin mit der schnöden Frage: „Sie wollen doch eine Bekanntschaft?“38 Die Bemerkung zerstörte nicht nur die Gefühlsgrundlage, sondern denunzierte auch das vergangene briefliche Phantasiespiel als Fälschung - jedenfalls von Seiten Wollheims. Das war nicht zu reparieren, der Briefwechsel, das Freundschaftsverhältnis zerbrechen.

Jetzt begreifen wir, was Nietzsche mit dem „Punsch der Briefstimmung“ meinte. Es ist jener Enthusiasmus, der sich an den eigenen Gefühlen so berauscht, dass er Einstimmigkeit suggeriert, wo zumindest untergründig Unstimmigkeit herrscht. Die Gefahr des Missverständnisses ist groß, weil die Selbsterregung in der Schreibsituation selber kein Korrektiv findet. Dass darin aber auch eine große Chance liegt, demonstrieren selbst die zuletzt misslungenen Briefınszenierungen: sie widersprechen der Tatsachenrealität, halten sich an die Möglichkeit des Gelingens.

Wie immer man die Briefkultur in ihre einzelnen Ausprägungen zerlegt, zuletzt wird man selbst der Informationsmitteilung ein Moment der Unsicherheit nicht absprechen können, das objektiv im Prinzip der Wahrscheinlichkeit, subjektiv in der unvorhersehbaren Wirkung auf den Adressaten begründet ist. Wie das wirkliche Gespräch ist auch das Brief - Gespräch eine Kunstform des Erprobens, ein Laboratorium des Möglichen, und hat ihm gegenüber dadurch einen Raum größerer Freiheit voraus, in dem wir leichter ausspielen können, was wir uns wünschen, als dies von Angesicht zu Angesicht opportun oder erlaubt wäre.

Dass er bis heute Konventionen und Mustern folgt, die rhetorischen Ursprungs sind, ist gerade eine Bedingung dieser Freiheit. Sie wirken nicht einschränkend, sondern wie Grenzpfähle gegen die Zwänge des alltäglichen Lebens.

Und heute? Private Briefe auf Papier und per Post befördert schreiben immer weniger Korrespondenten, das gilt selbst für Geschäftsbriefe. Die elektronischen Medien haben das Briefgespräch verändert, nicht weil der Bildschirm an die Stelle des Papiers getreten ist, sondern weil der zeitliche Abstand zwischen Brief und Antwortbrief extrem eingezogen wird und es nur an Präsenz und Willen des Adressaten liegt, ihn im Moment des Absendens zu lesen und sogleich zu beantworten. Die Gesprächssequenzen können einander also beinah wie im wirklichen Gespräch auf dem Fuße folgen, beim Chatten ist im zeitlichen Rhythmus der Unterschied in dieser Hinsicht völlig zu vernachlässigen. Das hat sogar Folgen für die räumliche Distanz: man empfındet das Entfernsein des anderen nicht mehr in dem Maße wie beim herkömmlichen Brief. Denn der andere kann sofort intervenieren, wir erfahren seine Stimmung und Gefühle sogleich als ob er neben uns stünde, er ist präsent, wenn auch nicht in seiner körperlichen

38. Ibid., S. 155. 
Erscheinung. So kommt es, dass die elektronische Kommunikation nun auch alles erlaubt, „was im Umgange erlaubt ist“, wovor Gellert das Briefgespräch bewahrt sah. ${ }^{39}$ Maskierungen, Rollenspiele sind deshalb nicht verschwunden, aber reduziert auf die pragmatische Funktion, nicht in seiner wahren Identität erkannt zu werden. Im übrigen reihen sich die elektronisch geführten Gespräche beinah gänzlich in die Alltagskommunikation ein, Regeln, die darüber hinaus gingen, sind nicht zu erkennen. Die Form, das Prinzip jeder Kultur, ist, wenn nicht ganz der Formlosigkeit gewichen, so doch einem anything goes, das in Anrede, Inhalt, Wortschatz und Stil nur noch eine gewisse Abhängigkeit von Trends und Moden zeigt.

39. Gellert, Die epistolographischen Schriften, S. 3. 